\title{
The research of technological properties of the titanium alloy Ti-6Al-4V. Part 1. Microstructure and mechanical properties
} \author{
R.M. Galeyev ${ }^{1}$, O.R. Valiakhmetov ${ }^{1}$ \\ †dr_rvs@mail.ru
}

R.V. Safiullin ${ }^{1, \dagger}$, A.R. Safiullin', S.P. Malysheva ${ }^{1}$, A.N. Kozlov², A.V. Berestov ${ }^{2}$,

${ }^{1}$ Institute for Metals Superplasticity Problems Russian Academy of Sciences, Khalturin str. 39, Ufa, 450001, Russia ${ }^{2}$ PSC VSMPO-AVISMA CORPORATION Parkovaya St. 1, Verkhnaya Salda, Sverdlovsk Region, 624760, Russia

The paper describes the results of comprehensive studies of the microstructure, mechanical and technological properties (formability and weldability in the solid state) of the titanium sheet alloy VT6 (Ti-6Al-4V) with improved superplastic properties produced by PSC «VSMPO-AVISMA». In the first part the initial microstructure of the alloy and its mechanical properties over a wide temperature range from 650 to $900^{\circ} \mathrm{C}$ at strain rates of $4 \times 10^{-4}, 4 \times 10^{-3}$ and $4 \times 10^{-2} \mathrm{~s}^{-1}$ have been investigated. It is found that the initial microstructure of the sheet is uniform and ultrafine grained with an average size of $1.2 \mu \mathrm{m}$. The initial grain size of the microstructure is varied from 0.1 to 4 microns. Most of the grains have a size from 0.5 to 1.5 microns. Mechanical tensile tests revealed that the ultrafine grained titanium alloy VT6 possesses higher superplastic characteristics as compared with those of the standard VT6 sheet. The microstructure of deformed samples and data on changes in the grain size depending on the temperature and strain rate are investigated and represented. The comparison of the superplastic characteristics of the samples cut along and across to the rolling direction indicates the absence of anisotropy. The studied alloy exhibits the best superplastic properties at temperatures ranging from 700 to $850^{\circ} \mathrm{C}$ and strain rates of $4 \times 10^{-4}-4 \times 10^{-3} \mathrm{~s}^{-1}$, which corresponds to the manifestation of the low-temperature superplasticity. Superplastic elongations are from 650 to $1075 \%$. That allows us to recommend the produced sheet alloy for development of low temperature processing methods based on superplastic forming and diffusion welding to manufacture the parts for aerospace industry.

Keywords: two-phase titanium alloy VT6, microstructure, flow stresses, low-temperature superplasticity, superplastic forming.

\section{Исследование технологических свойств титанового сплава}

\section{Ti-6Al-4V. Часть 1. Микроструктура и механические свойства}

\author{
Сафиуллин Р.В. ${ }^{1, \dagger}$, Сафиуллин А.Р. ${ }^{1}$, Малышева С.П. ${ }^{1}$, Козлов А.Н. ${ }^{2}$, Берестов А.В. ${ }^{2}$, \\ Галеев Р.М. ${ }^{1}$, Валиахметов О.Р. ${ }^{1}$ \\ †dr_rvs@mail.ru
}

${ }^{1}$ Институт проблем сверхпластичности металлов РАН, ул. Ст. Халтурина 39, 450001 г. Уфа, Россия

${ }^{2}$ ПАО «Корпорация ВСМПО-АВИСМА», ул. Парковая 1, 624760, Верхняя Салда, Россия

В работе описаны результаты комплексных исследований микроструктуры, механических и технологических свойств (формуемость и свариваемость в твердом состоянии) нового листового титанового сплава BT6 (Ti-6Al-4V) с улучшенными сверхпластическими свойствами производства ПАО «Корпорация ВСМПО-АВИСМА». В первой части статьи подробно исследована исходная микроструктура данного сплава и его механические свойства в широком диапазоне температур от 650 до $900^{\circ} \mathrm{C}$ при скоростях деформации $4 \times 10^{-4}, 4 \times 10^{-3}, 4 \times 10^{-2} \mathrm{c}^{-1}$. Установлено, что исходная микроструктура однородная, ультрамелкозернистая со средним размером зерен равным 1,2 мкм. В исходной микроструктуре встречаются зерна размером от 0,1 до 4 мкм. Большинство зерен имеет размер от 0,5 до 1,5 мкм. Механические испытания на растяжение позволили установить, что титановый сплав ВТ6 с ультрамелкозернистой микроструктурой обладает более высокими характеристиками сверхпластичности по сравнению со стандартным листом. Проведены исследования микроструктуры деформированных образцов и приведены данные по изменению размеров зерен в зависимости от температуры и скорости деформации. Сравнение характеристик сверхпластичности образцов, вырезанных вдоль и поперек направления прокатки, свидетельствует об отсутствии анизотропии. 
Наилучшие сверхпластические свойства данный сплав проявляет при температурах от 700 до $850^{\circ} \mathrm{C}$ и скоростях деформации $4 \times 10^{-4}-4 \times 10^{-3} \mathrm{c}^{-1}$, что соответствует проявлению низкотемпературной сверхпластичности. Относительное удлинение при этом составляет от 650 до 1075\%, что позволяет рекомендовать данный сплав для разработки низкотемпературных технологических процессов сверхпластической формовки и диффузионной сварки для получения изделий авиакосмической техники.

Ключевые слова: двухфазный титановый сплав ВТ6, микроструктура, напряжения течения, низкотемпературная сверхпластичность, сверхпластическая формовка.

\section{1. Введение}

Успех внедрения технологии сверхпластической формовки (СПФ) и сверхпластической формовки в сочетании со сваркой давлением (СПФ/СД) в производство во многом зависит от проведения систематических исследований сверхпластичных материалов, изучения их механических и технологических свойств и их поведения в условиях сверхпластической деформации. Наряду с известными преимуществами процесса СПФ/СД по сравнению с традиционными технологиями у данного метода имеются ограничения и недостатки. Прежде всего, это высокие рабочие температуры процесса, приводящие к использованию дорогостоящей жаропрочной оснастки и к образованию хрупкого альфированного слоя на поверхности получаемых из титановых сплавов изделий. В этой связи снижение температуры деформирования является одной из важнейших задач для процесса СПФ/СД. Снижение температуры сверхпластической обработки возможно при использовании материалов, имеющих ультрамелкозернистую, субмикрокристаллическую или нанокристаллическую структуру [1-3]. Однако, известно очень мало работ, посвященных исследованию таких материалов для использования в машиностроении и авиакосмической техники. В работах $[1,4$ - 7] проведено систематическое исследование технологических свойств листовых заготовок из титанового сплава ВТ6 с субмикрокристаллической (СМК) структурой. Показано, что данные листовые заготовки обладают хорошей формуемостью и свариваемостью в твердом состоянии в условиях низкотемпературной сверхпластичности и могут быть успешно использованы в технологии СПФ/СД. В работе [1] показано, что использование титанового сплава с СМК структурой позволяет снизить температуру сверхпластической (СП) деформации на $200^{\circ} \mathrm{C}$ по сравнению с деформацией материала с микрокристаллической структурой, при этом и механические свойства сплава с СМК структурой на 20...30\% выше, чем у обычного промышленного сплава. Основные преимущества, получаемые при снижении температуры процесса СПФ/СД, являются значительное повышение стойкости штампов, уменьшение энергозатрат на нагрев и изготовление деталей, и соответственно сокращение технологического цикла их изготовления. Специалисты российского предприятия ПАО «Корпорация ВСМПОАВИСМА» разработали новое поколение листового титанового сплава ВТ6 (Ti-6Al-4V) с улучшенными сверхпластическими характеристиками и ультрамелкозернистой структурой специально для процесса СПФ при пониженных температурах. В работах специалистов фирм Boeing и Aeromet [8-10] приведены результаты исследований данного сплава и возможности использования его в процессах СПФ. Отмечается, что сплав может успешно применяться для изготовления полых листовых конструкций методами СПФ и СПФ/СД при температуре $775^{\circ} \mathrm{C}$. Однако систематических исследований данного листового титанового сплава проведено не было. В этой связи целью настоящей работы являются комплексные исследования микроструктуры, механических свойств, формуемости и свариваемости в твердом состоянии серийного листового титанового сплава ВТ6 производства ПАО «Корпорация ВСМПОАВИСМА» с ультрамелкозернистой структурой и возможного его использования в технологии СПФ/СД.

\section{2. Материал и методика исследования}

В качестве материала исследования был использован листовой титановый сплав ВT6 (Ti-6Al-4V) толщиной 1 мм производства ПАО «Корпорация ВСМПО-АВИСМА». Исследование микроструктуры исходных образцов и образцов после механических испытаний проводили с помощью оптических микроскопов «Olympus GX51» и «Axiovert100» при увеличениях 100-1000, сканирующего электронного микроскопа TESCAN при увеличениях 25-5000 и просвечивающего микроскопа JEOL-2000ЕХ. Всего исследовано более 100 полей зрения. Размер зерен оценивался по темнопольным изображениям. Исследования микроструктуры листов проводили в трех взаимно перпендикулярных плоскостях листа, а также в головках и рабочей части (шейке) деформированных образцов. Механические свойства при растяжении определяли на плоских образцах сечением 5 мм $\times 1$ мм и длиной рабочей части 20 мм. Образцы для исследования вырезали из листов в двух направлениях относительно направления прокатки (вдоль и поперек прокатки). На каждую точку было использовано по 3 образца. Испытания на растяжение проводили на машине “INSTRON" модели 1185 при температурах $650,700,750,800,850$ и $900^{\circ} \mathrm{C}$ в воздушной атмосфере, начальные скорости деформации были $4 \times 10^{-4}, 4 \times 10^{-3}$ и $4 \times 10^{-2} \mathrm{c}^{-1}$. Для защиты от газонасыщения и окисления применяли эмалевые покрытия марки ЭВТ24 и ЭВТ26, которые наносили на рабочую часть плоского образца. При испытаниях перепад температуры в 3-х секционной печи нагрева на длине 150 мм был не более $\pm 5^{\circ} \mathrm{C}$. По результатам механических испытаний определяли напряжения течения, относительное удлинение и коэффициент скоростной чувствительности «m». Полученные результаты сравнивали с механическими свойствами листов толщиной 1,5 мм производства ПАО «Корпорация ВСМПО-АВИСМА», полученных прокаткой по обычной серийной технологии. 


\section{3. Результаты и их обсуждение}

На рис. 1 - 2 приведена микроструктура листа толщиной 1 мм в трех взаимно перпендикулярных сечениях $(X-$ плоскость прокатки листа, $Y$ и $Z-$ плоскости поперек к плоскости прокатки). Видно, что во всех сечениях листа структура однородная, зерна равноосные. Лишь в некоторых участках в плоскости прокатки выявляются вытянутые зерна. В сечении $Z$ зерна немного вытянуты в направлении прокатки. В сечении $Y$ встречается строчечность - цепочки более мелких зерен. Объемная доля $\beta$-фазы в плоскостях $X, Y$ и $Z$ составляет, соответственно, 19,16 и $19 \%$.

На рис. 3,a,b приведена микроструктура листа из сплава ВТ6 в плоскости прокатки $X$, полученная с помощью просвечивающего электронного микроскопа. Видно, что микроструктура однородная, равноосные микрофрагменты и зерна имеют средний размер 1,2 мкм, четкие границы зерен отсутствуют. В образце имеются значительные внутренние напряжения. На рис. 3 в приведена гистограмма распределений зерен по размерам в листе из сплава ВT6. Размер зерен изменяется от 0,1 до 4 мкм.

На рис. 4 представлены образцы, вырезанные из листов толщиной 1 и 1,5 мм, деформированные при различных температурно-скоростных условиях.
В таблице 1 приведены результаты механических испытаний при повышенных температурах образцов, вырезанных из листов толщиной 1 и 1,5 мм. Анализируя данные таблицы 1 , необходимо отметить, что у обоих листов при всех температурах испытаний напряжения течения возрастают с повышением скорости деформации и понижением температуры. Вместе с тем, зависимость относительного удлинения от температурно-скоростных условий носит несколько иной характер.

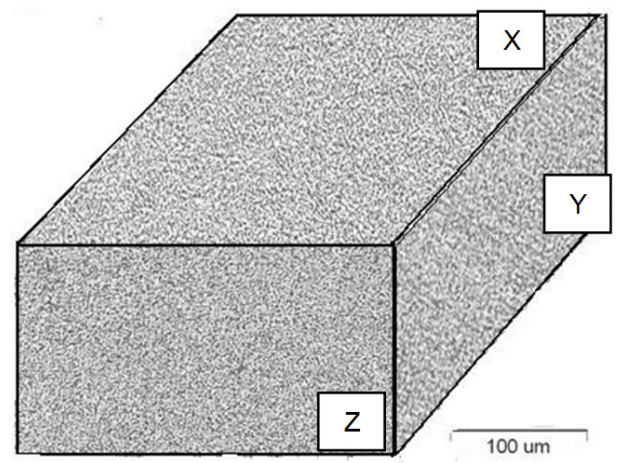

Рис. 1. Микроструктура листа из сплава ВТ6 в трех взаимно перпендикулярных направлениях (оптическая микроскопия).

Fig. 1. The microstructure of the alloy VT6 sheet in three mutually perpendicular directions (optical microscopy).

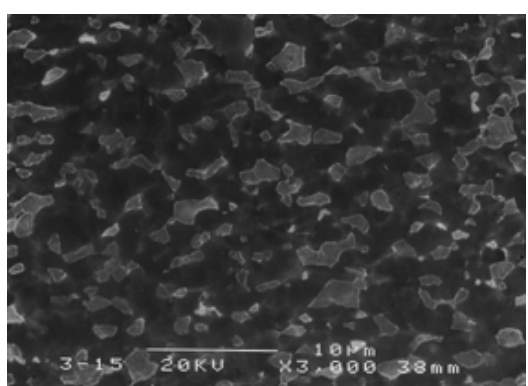

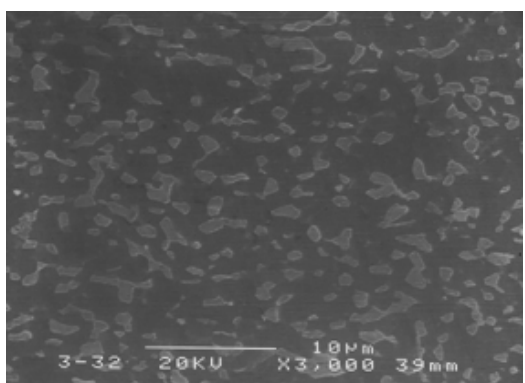

b

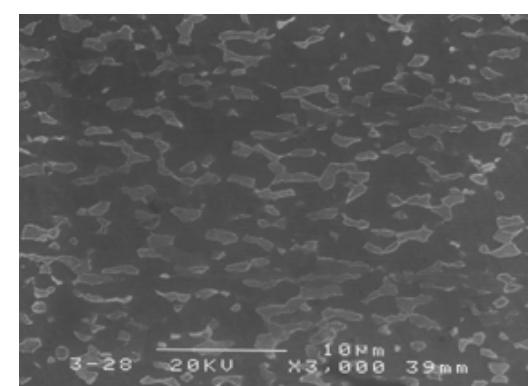

Рис. 2. Микроструктура листа в различных сечениях: (а) - плоскость $X$, (b) - плоскость $Y$, (c) - плоскость $Z$ (растровая электронная микроскопия).

Fig. 2. The microstructure of the sheet in the different sections: (a) - the plane $X$, (b) - the plane $Y$, (c) - the plane $Z$ (scanning electron microscopy).

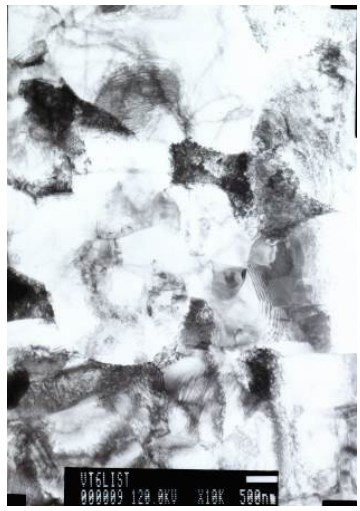

a

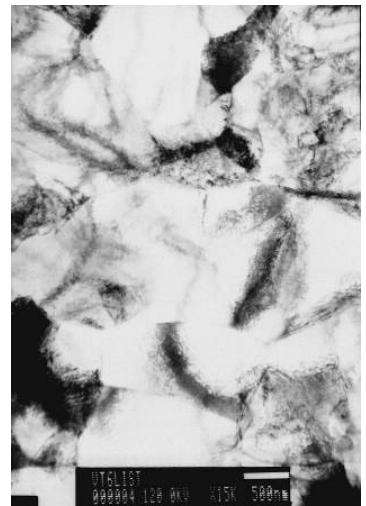

b

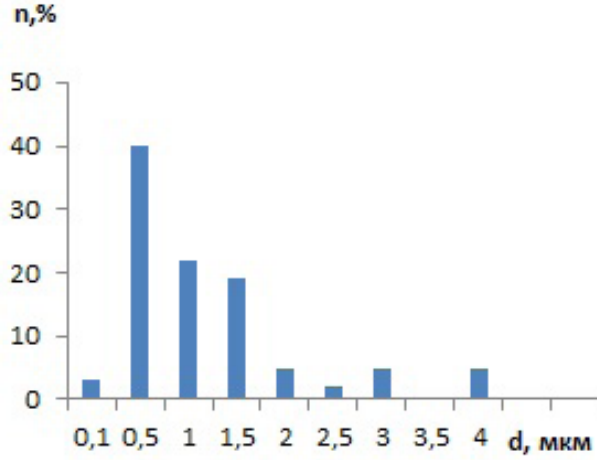

Рис. 3. Микроструктура листа из сплава ВТ6: (a), (b) - просвечивающая электронная микроскопия, (c) - гистограмма распределений зерен по размерам в листе из сплава ВТ6.

Fig. 3. The microstructure of the alloy VT6 sheet ( $(, b)$ (transmission electron microscopy), (c) - histogram grain size distribution in the sheet alloy VT6. 
В листе толщиной 1,5 мм относительное удлинение растет с повышением температуры и снижением скорости деформации. В листе толщиной 1 мм относительное удлинение растет с увеличением температуры до $700^{\circ} \mathrm{C}$, а при дальнейшем увеличении температуры удлинение практически постоянно. Уменьшение скорости деформации при температурах от 650 до $700^{\circ} \mathrm{C}$ приводит к росту пластичности, а при $750-900^{\circ} \mathrm{C}$ относительное удлинение имеет максимальные значения при скорости деформации $4 \times 10^{-3} \mathrm{c}^{-1}$. Вероятно, такое поведение материала обусловлено влиянием исходной микроструктуры листов. Лист толщиной 1 мм имеет микроструктуру с размером зерен 1,2 мкм, которая стабильна для температур менее $750^{\circ} \mathrm{C}$, а при нагреве выше $750^{\circ} \mathrm{C}$ в листе происходит интенсивный рост зерен. Кроме того, при горячей деформации уменьшение скорости деформации влечет за собой также рост зерен, что приводит к снижению пластичности.

Значения размеров зерен испытанных образцов, вырезанных из листа толщиной 1 мм, представлены в таблице 2. Видно, что размер зерен в головках образцов, деформированных при температурах 650 и $700^{\circ} \mathrm{C}$, не изменяется, что говорит о термостабильности исходной микроструктуры при этих температурах, причем с уменьшением скорости деформации увеличивается время выдержки материала при температуре испытания. В рабочей части (шейке) образцов структурные изменения более значительны. При $650^{\circ} \mathrm{C}$ и скоростях деформации $4 \times 10^{-4} \mathrm{c}^{-1}$ и $4 \times 10^{-3} \mathrm{c}^{-1}$ размер зерен практически не меняется, а при скорости деформации $4 \times 10^{-2} \mathrm{c}^{-1}$ наблюдается уменьшение размера зерен; уже при температуре $700^{\circ} \mathrm{C}$ наблюдается рост зерен, причем с уменьшением скорости деформации рост более интенсивный.

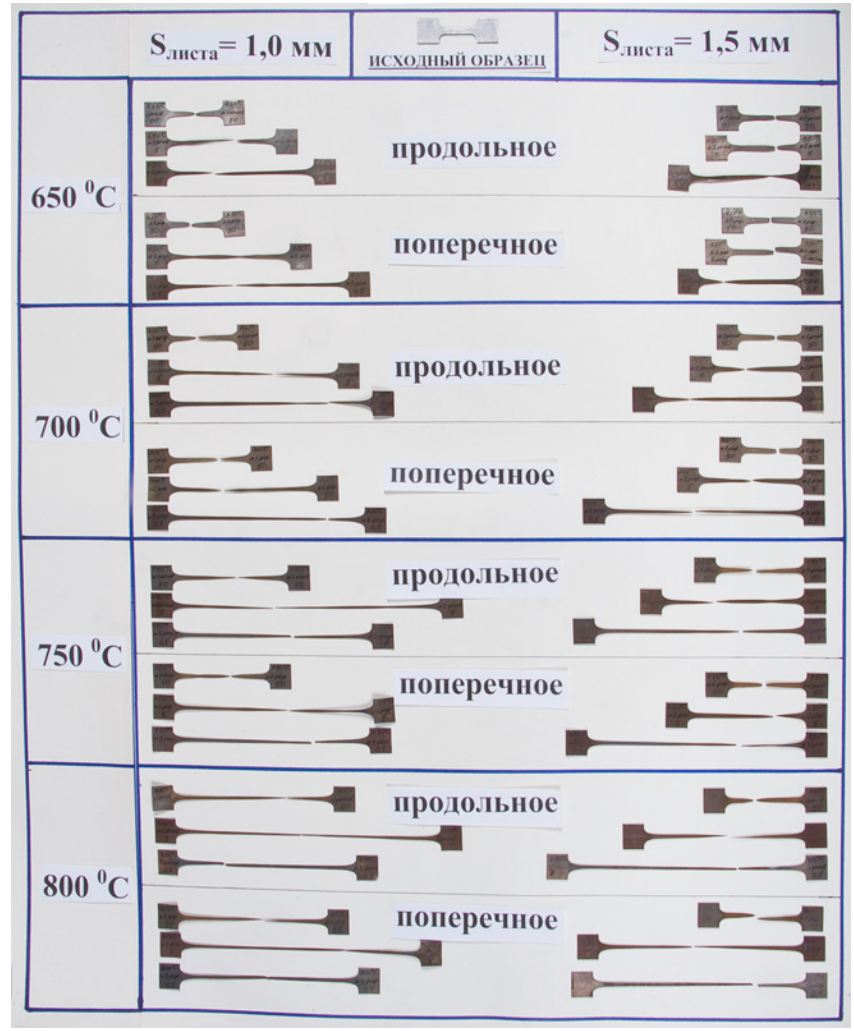

Рис. 4. Образцы из титанового сплава ВТ6 после испытаний на растяжение при повышенных температурах.

Fig. 4. Samples of titanium alloy VT6 after the tensile tests at elevated temperatures.

Табл. 1. Механические свойства листов толщиной 1 и 1,5 мм из титанового сплава ВТ6.

Table 1. Mechanical properties of the sheets with thickness of 1 and $1.5 \mathrm{~mm}$ out of titanium alloy VT6.

\begin{tabular}{|c|c|c|c|c|c|c|c|c|c|c|c|c|c|}
\hline \multirow{3}{*}{$\begin{array}{l}\mathrm{T} \\
{ }^{\circ} \mathrm{C}\end{array}$} & \multirow{3}{*}{$\begin{array}{l}\dot{\varepsilon} \\
\mathrm{s}^{-1}\end{array}$} & & & \multicolumn{2}{|c|}{$\begin{array}{c}\sigma, \mathrm{MPa} \\
(1,5 \mathrm{~mm})\end{array}$} & \multicolumn{2}{|c|}{$\begin{array}{c}\delta, \% \\
(1 \mathrm{~mm})\end{array}$} & \multicolumn{2}{|c|}{$\begin{array}{c}\delta, \% \\
(1,5 \mathrm{~mm})\end{array}$} & \multicolumn{2}{|c|}{$\begin{array}{c}m \\
(1 \mathrm{~mm})\end{array}$} & \multicolumn{2}{|c|}{$\begin{array}{c}m \\
(1,5 \mathrm{~mm})\end{array}$} \\
\hline & & \multicolumn{12}{|c|}{$\begin{array}{l}\text { Угол вырезки образца к направлению прокатки листа } \\
\text { The angle of cutting the sample to the direction of sheet rolling }\end{array}$} \\
\hline & & $0^{\circ}$ & $90^{\circ}$ & $0^{\circ}$ & $90^{\circ}$ & $0^{\circ}$ & $90^{\circ}$ & $0^{\circ}$ & $90^{\circ}$ & $0^{\circ}$ & $90^{\circ}$ & $0^{\circ}$ & $90^{\circ}$ \\
\hline \multirow{3}{*}{650} & $4 \times 10^{-4}$ & 145 & 130 & 240 & 233 & 450 & 560 & 310 & 265 & 0,31 & 0,32 & 0,22 & 0,23 \\
\hline & $4 \times 10^{-3}$ & 320 & 280 & 433 & 392 & 295 & 360 & 135 & 150 & 0,25 & 0,26 & 0,20 & 0,21 \\
\hline & $4 \times 10^{-2}$ & 500 & 475 & 550 & 517 & 85 & 85 & 100 & 75 & 0,10 & 0,10 & 0,11 & 0,10 \\
\hline \multirow{3}{*}{700} & $4 \times 10^{-4}$ & 60 & 63 & 127 & 133 & 675 & 650 & 450 & 640 & 0,36 & 0,35 & 0,33 & 0,33 \\
\hline & $4 \times 10^{-3}$ & 190 & 200 & 308 & 283 & 525 & 450 & 210 & 260 & 0,32 & 0,31 & 0,25 & 0,27 \\
\hline & $4 \times 10^{-2}$ & 390 & 375 & 467 & 417 & 125 & 175 & 100 & 90 & 0,21 & 0,22 & 0,15 & 0,16 \\
\hline \multirow{3}{*}{750} & $4 \times 10^{-4}$ & 25 & 30 & 62 & 59 & 650 & 650 & 675 & 725 & 0,36 & 0,36 & 0,36 & 0,37 \\
\hline & $4 \times 10^{-3}$ & 100 & 105 & 192 & 195 & 950 & 675 & 415 & 325 & 0,42 & 0,38 & 0,33 & 0,32 \\
\hline & $4 \times 10^{-2}$ & 275 & 270 & 350 & 317 & 325 & 250 & 200 & 165 & 0,30 & 0,28 & 0,25 & 0,22 \\
\hline \multirow{3}{*}{800} & $4 \times 10^{-4}$ & 10 & 9,5 & 38 & 40 & 600 & 600 & 800 & 700 & 0,35 & 0,35 & 0,38 & 0,37 \\
\hline & $4 \times 10^{-3}$ & 55 & 55 & 113 & 105 & 950 & 850 & 500 & 685 & 0,45 & 0,43 & 0,35 & 0,38 \\
\hline & $4 \times 10^{-2}$ & 180 & 180 & 255 & 247 & 525 & 475 & 170 & 180 & 0,33 & 0,30 & 0,21 & 0,22 \\
\hline \multirow{3}{*}{850} & $4 \times 10^{-4}$ & 5,8 & 5,2 & - & - & 800 & 770 & - & - & 0,9 & 0,9 & - & - \\
\hline & $4 \times 10^{-3}$ & 27 & 26 & - & - & 1075 & 925 & - & - & 0,8 & 0,9 & - & - \\
\hline & $4 \times 10^{-2}$ & 80 & 106 & - & - & 620 & 170 & - & - & 0,6 & 0,5 & - & - \\
\hline \multirow{3}{*}{900} & $4 \times 10^{-4}$ & 1,1 & 1,1 & - & - & 575 & 550 & - & - & 0,9 & 0,9 & - & - \\
\hline & $4 \times 10^{-3}$ & 15 & 14 & - & - & 675 & 655 & - & - & 0,8 & 0,8 & - & - \\
\hline & $4 \times 10^{-2}$ & 64 & 66 & - & - & 205 & 175 & - & - & 0,48 & 0,42 & - & - \\
\hline
\end{tabular}


Табл. 2. Размер зерен (мкм) в деформированных образцах титанового сплава ВТ6 (лист толщиной 1 мм).

Table 2. The grain size $(\mu \mathrm{m})$ in the deformed samples of titanium alloy VT6 (sheet thickness of $1 \mathrm{~mm}$ ).

\begin{tabular}{|c|c|c|c|c|c|c|}
\hline \multirow{2}{*}{$T,{ }^{\circ} \mathrm{C}$} & \multicolumn{5}{|c|}{ Скорость деформации/Strain rate } \\
\cline { 2 - 7 } & \multicolumn{2}{|c|}{$4 \times 10^{-4} \mathrm{~s}^{-1}$} & \multicolumn{2}{c|}{$4 \times 10^{-3} \mathrm{~s}^{-1}$} & \multicolumn{2}{c|}{$4 \times 10^{-2} \mathrm{~s}^{-1}$} \\
\hline & головка/head & шейка/neck & головка/head & шейка/neck & головка/head & шейка/neck \\
\hline 650 & 1,5 & 1,5 & 1,5 & 1,5 & 1,5 & 1,0 \\
\hline 700 & 1,5 & 4,0 & 1,5 & 2,5 & 1,5 & 1,5 \\
\hline 750 & 2,0 & 5,0 & 1,8 & 3,0 & 2,3 & 2,0 \\
\hline 800 & 4,0 & 7,5 & 2,5 & 5,0 & 2,9 & 3,4 \\
\hline 850 & 5,8 & 8,2 & 4,6 & 6,3 & 3,9 & 4,7 \\
\hline 900 & 8,8 & 12,4 & 5,1 & 6,8 & & \\
\hline
\end{tabular}

При температурах 750 и $800^{\circ} \mathrm{C}$ отмечается рост зерен как в головках, так и рабочей части образцов, причем, с уменьшением скорости деформации (увеличении времени выдержки), рост зерен идет сильнее. Особенно это видно при температуре $800^{\circ} \mathrm{C}$, что приводит к снижению пластичности материала.

Лист толщиной 1,5 мм имеет больший размер зерен, и его микроструктура стабильна в исследованном температурном интервале, поэтому механическое поведение этих листов различается.

Проведенные исследования (таблица 1) не выявили существенной анизотропии в механических свойствах листов обоих типов, что весьма благоприятно для сверхпластической формовки.

Из данных испытаний (таблица 1) видно, что в исследованном температурно-скоростном интервале деформации листы из сплава ВТ6 имеют высокие значения скоростной чувствительности напряжения течения к скорости деформации (коэффициент $m$ ), что свидетельствует о сверхпластическом течении материала. На это указывает также равномерная деформация рабочей части образцов, испытанных в оптимальных скоростных условиях сверхпластической деформации $\left(4 \times 10^{-3}-4 \times 10^{-4} \mathrm{c}^{-1}\right)$ (рис. 4). Вместе с тем, в листах толщиной 1 мм с ультрамелкозернистой структурой наблюдается снижение температуры проявления сверхпластической деформации на $200-250^{\circ} \mathrm{C}$ по сравнению со сплавом ВТ6 в обычном состоянии [11]. Лист толщиной 1 мм имеет более высокие характеристики сверхпластичности (меньшие напряжения течения, большие относительные удлинения) по сравнению с листом толщиной 1,5 мм, которые сохраняются до $800^{\circ} \mathrm{C}$.

\section{4. Заключение}

Проведены исследования микроструктуры и механических свойств листового титанового сплава ВТ6 производства ПАО «Корпорация ВСМПО-АВИСМА» при температурах $650-900^{\circ} \mathrm{C}$. Показано, что в исходном состоянии листы имеют однородную ультрамелкозернистую структуру со средним размером зерен 1,2 мкм. Исследование механических свойств на растяжение данных листов показалои, что наилучшие сверхпластические свойства отмечаются в температурном диапазоне от 700 до $850^{\circ} \mathrm{C}$. Анизотропия механических свойств в листах не наблюдается, что весьма благоприятно для сверхпластической формовки.

\section{Литература/References}

1. O. A. Kaibyshev, R. V. Safiullin, R. Ya. Lutfullin, O. R. Valiakhmetov, R. M. Galeyev, A. Dutta, T. Raghu, G. G. Saha, Journal Materials Science and Technology, 22 (3), 343 - 348 (2006).

2. G.A. Salishchev, R.M. Galeyev, S.P. Malysheva, O.R. Valiakhmetov: Mater. Sci. Forum, 585, $243-245$ (1997).

3. G.A. Salishchev, R.M. Galeyev, O.R. Valiakhmetov, R. V. Safiullin, R. Ya. Lutfullin, O.N. Senkov, F. H. Froes and O.A. Kaibyshev: J. Mater. Process. Technol. 116, 265-268, (2001).

4. O.A. Rudenko, A.A. Kruglov, R.V. Safiullin, O.R. Valiakhmetov, R. Ya. Lutfullin Kuznechnoshtampovochnoe proizvodctvo 4, 5 (2006). (In Russian) [О.А. Руденко, А.А. Круглов, Р.В. Сафиуллин, О.Р. Валиахметов, Р.Я. Лутфуллин Кузнечноштамповочное производство 4, 5, (2006)].

5. R. V. Safiullin, W. Beck, A. R. Safiullin, M. A. Murzinova Proceedings of the 6th Europe Conference on Superplastic Forming, (2008), 14 pages. http://hal.archives-ouvertes.fr/hal--00348660/fr ([hal$-00348660 /$ fr-version 1]).

6. R. V. Safiullin. In Proceedings of Third European Conference on Superplastic Forming. (2004) p.167 - 174.

7. E.N. Petrov V.V. Rodionov, E.N. Kuzmin, R. Ya. Lutfullin, R. V. Safiullin. Honeycomb. Sneginsk (2008) 176 p. (In Russian) [Е.Н. Петров, В.В. Родионов, Э.Н. Кузьмин, Р.Я. Лутфуллин, P.В. Сафиуллин. Ячеистые конструкции. (ISBN 978-5-902278-26-9) Снежинск: Издательство РФЯЦ-ВНИИТФ. (2008) 176 с.]

8. P. N. Comley. Materials Science Forum 233, 447-448 (2004).

9. L.D. Hefti. Journal of Materials Engineering and Performance, 178, 178 - 182 (2008).

10. B. Swale. Proceedings of the Europe Conference on Superplastic Forming "Euro-SPF 2004” p.39-44 (2004).

11. O.A. Kaibyshev F.Z. Utyashev. Superplasticity, structure refinement and processing of hardalloy. Moscow, Science. (2002) 440 p. (In Russian) [О.А. Кайбышев, Ф. З. Утяшев. Сверхпластичность, измельчение структуры и обработка труднодеформируемых сплавов М: Наука, 2002. 440 с.]. 